\title{
Timing of antibiotics, volume and vasoactive infusions in children with sepsis: it is all in the timing
}

\author{
Samiran Ray ${ }^{1}$ and Mark J. Peters ${ }^{1,2^{*}}$ \\ See related research by van Paridon et al., http://www.ccforum.com/content/19/1/293
}

We share the view of van Paridon et al. [1] that robust data on fluid bolus therapy for sepsis resuscitation are needed, and looked with interest to the Alberta Sepsis Network's recent report to add to our understanding. We note that the authors chose to exclude children who were expected to die $<24$ hours from presentation. We would be grateful to know how many cases were excluded on this basis. The majority of the hazard from sepsis lies in this period. We recently defined this risk in a similar cohort of children; $55 \%$ of all sepsis deaths (78\% of these being previously healthy children) occurred within the first 24 hours [2].

Resuscitation interventions such as fluid and vasoactive drugs are guided by changes in physiology. Inasmuch as physiological variables are associated with mortality, these interventions are also likely to have an immediate effect on mortality. The authors suggest potential explanations for the 1-year mortality effect seen with aggressive fluid administration: while all are not only plausible but likely, the effect will only be seen if the acute phase is overcome. By excluding children dying early, the authors also reduce the potential to propensity match children receiving similar levels of interventions. Pediatric Risk of Mortality (PRISM) scores control for severity of illness, but information is lost given that the PRISM score utilises categories of haemodynamic variables rather than age-standardised continuous variables [3]. We wonder whether the modest effect size difference would persist with these alterations to the methodology.

\section{Authors' response}

Bregje M van Paridon, Cathy Sheppard, Gonzalo Garcia Guerra and Ari R Joffe

We thank Ray and Peters for their interest in our study. They point out that we excluded children who were expected to die within 24 hours from presentation, and that these are the children who may be most likely to benefit from aggressive fluid resuscitation. They hypothesise that aggressive fluid administration may be non-beneficial only once the 'acute phase' is overcome.

We agree this is a limitation of our study. In our cohort of children screened for sepsis in the paediatric ICU, one child was excluded because survival for $>24$ hours was not expected and one child was excluded because palliative care had been decided upon. Although we do not have exact numbers, the number of children with sepsis

\footnotetext{
* Correspondence: mark.peters@ucl.ac.uk

'Respiratory, Critical Care and Anaesthesia Section, UCL Institute of Child Health, 30 Guildford Street, London WC1N 1EH, UK

${ }^{2}$ Paediatric and Neonatal Intensive Care Unit, Great Ormond Street Hospital, London WC1N 3JH, UK
}

presenting to our Children's Hospital emergency department or referral area and dying before arriving in the paediatric ICU is extremely small. In addition, although only one patient in our cohort died in the paediatric ICU, six children required ECLS (extracorporeal life-support), an indicator of the severity of sepsis [4]. Thus, we believe the number of patients excluded from our study who died within 24 hours after sepsis presentation is very small. In addition, we note that recent editorials continue to point out the variability in fluid bolus therapy practice, the limited data on effectiveness of fluid bolus therapy and that the ideal resuscitation target is a knowledge gap in sepsis [5-7]. Although we do not recommend a change in practice at this time, we believe that further study of fluid bolus therapy and alternative strategies for resuscitation in sepsis are warranted.

\section{Abbreviations}

PRISM: Pediatric Risk of Mortality. 


\section{Competing interests}

The authors declare that they have no competing interests.

\section{Published online: 21 November 2015}

\section{References}

1. van Paridon BM, Sheppard C, Guerra GG, Joffe AR, for the Alberta Sepsis Network. Timing of antibiotics, volume, and vasoactive infusions in children with sepsis admitted to intensive care. Crit Care. 2015;19:293.

2. Cvetkovic M, Lutman D, Ramnarayan P, Pathan N, Inwald DP, Peters MJ. Timing of death in children referred for intensive care with severe sepsis: implications for interventional studies. Pediatr Crit Care Med. 2015;16:410-7.

3. Tibby SM. Does PELOD measure organ dysfunction ... is organ function a valid surrogate for death? Intensive Care Med. 2010;36:4-7.

4. Mickiewicz B, Thompson G, Blackwood J, Jenne C, Winston B, Vogel H, et al. Development of metabolic and inflammatory mediator biomarker phenotyping for early diagnosis and triage of pediatric sepsis. Crit Care. 2015;19:320.

5. Gattas D, Aneman A. Consistent inconsistency of fluid challenges in the ICU: we should reflect on extreme variation in our practice. Intensive Care Med. 2015:4:1670-2.

6. Perner A, Vieillard-Baron A, Bakker J. Fluid resuscitation in ICU patients: quo vadis? Intensive Care Med. 2015;41:1667-9.

7. Seymour CW, Rosengart MR. Septic shock: advances in diagnosis and treatment. JAMA. 2015;314:707-17. 